\title{
Childhood adversity and inflammatory processes in youth: A prospective study
}

\author{
Natalie Slopen ${ }^{a, b, c,{ }^{*}, \text { Laura D. Kubzansky }}{ }^{b, 1}$, Katie A. McLaughlin ${ }^{a, d, e, 2}$, and Karestan C. \\ Koenen ${ }^{\dagger, 3}$ \\ aCenter on the Developing Child, Harvard University, United States \\ bHarvard School of Public Health, United States \\ 'Harvard Graduate School of Education, United States \\ dDivision of General Pediatrics, Children's Hospital Boston, United States \\ eHarvard Medical School, United States \\ ${ }^{f}$ Mailman School of Public Health, Columbia University, United States
}

\section{Summary}

Background-Retrospective studies show that childhood adversity is associated with systemic inflammation in adulthood. Few prospective studies have examined whether childhood adversity influences inflammation in an observable manner during childhood or adolescence and if these effects are sustained over time.

Methods-Using longitudinal data from the Avon Longitudinal Study of Parents and Children, we examined associations between acute adverse events at seven time points prior to age 8 and inflammation at ages 10 and 15. Inflammatory markers at age 10 included interleukin-6 (IL-6; $N=$ $4655)$ and C-reactive protein (CRP; $N=4647)$, and CRP was measured again at age 15 ( $N=$ 3286). We further evaluated whether body mass index (BMI), depression, or cigarette smoking mediated associations between adverse events and inflammation.

Results-Adverse events in middle childhood (occurring between ages 6 to 8), as well as cumulative adversity from birth to 8 years, were associated with higher levels of IL-6 and CRP at age 10 . Adverse events reported in early childhood (1.5 years) or middle childhood, and cumulative adversity from birth through 8 years predicted increased levels of CRP at age 15, and these associations persisted after adjustment for CRP at age 10. Some, but not all, of these associations were mediated by BMI.

Conclusions-This study documents that exposure to adverse events prior to age 8 is associated with elevated inflammation at age 10 and in mid-adolescence. These findings provide prospective evidence for a biological mechanism by which early experiences may shape long-term health. Future studies with earlier assessments of inflammation are necessary in order to elucidate

\footnotetext{
(C) 2012 Elsevier Ltd. All rights reserved.

"Corresponding author at: Center on the Developing Child, Harvard University, 50 Church Street, 4th Floor, Cambridge, MA 02138, United States. Tel.: +1 617733 0309; fax: +1 617496 1229., nslopen@hsph.harvard.edu (N. Slopen).

${ }_{1}^{1}$ Present address: Department of Society, Human Development, and Health, Harvard School of Public Health, 677 Huntington Avenue, Boston, MA 02115, United States.

2Present address: Division of General Pediatrics, Children's Hospital Boston, 21 Autumn Street, Boston, MA 02115, United States.

${ }^{3}$ Present address: Department of Epidemiology, Mailman School of Public Health, Columbia University, 722 West 168th Street, New York, NY 10032, United States.

Conflict of interest

None declared.
} 
potential sensitive periods and mechanisms that link childhood adversity to later disease vulnerability.

\section{Keywords}

Children; Adolescents; Inflammation; C-reactive protein; Interleukin-6; Acute stress; Stressful life events; Prospective cohort; Avon Longitudinal Study of Parents and Children (ALSPAC)

\section{Introduction}

Early life experiences have lasting effects on neurobiological functioning and health outcomes across the life course (Shonkoff et al., 2009; Taylor et al., 2011). Adverse childhood experiences — including low socioeconomic status (SES), maltreatment, or maladaptive early family environments - are associated with a broad spectrum of subsequent health problems in adulthood, including cardiovascular disorders (CVD) (Dong et al., 2004; Galobardes et al., 2006), metabolic abnormalities (Thomas et al., 2008; Danese et al., 2009), cancers (Felitti et al., 1998), arthritis (Dube et al., 2009; Von Korff et al., 2009) and mental illnesses (Chapman et al., 2004; Kessler et al., 2010). Evidence from both animal and human studies suggests that inflammation may be a central biological mechanism linking early adversity to a variety of health outcomes (Miller et al., 2009a). Although numerous studies show that low childhood SES and harsh early family environments are associated with elevated concentrations of inflammatory markers like circulating levels of C-reactive protein (CRP) and pro-inflammatory cytokines such as inter-leukin-6 (IL-6) among adults (Taylor et al., 2006; Danese et al., 2007; Pollitt et al., 2007), relatively few studies have examined whether childhood adversity is associated with inflammation during childhood or adolescence (Slopen et al., 2011). It is important to characterize the developmental progression of inflammatory processes in relation to adversity in children and adolescents in order to understand how exposure to childhood adversity may engender disease vulnerability over time (Danese et al., 2011). The aim of this study is to examine prospectively the relationship between adverse events that occur during childhood and inflammation in both childhood and adolescence.

Increasing evidence suggests that elevated levels of inflammation in childhood may provide an early marker of disease risk in adulthood. For example, elevated CRP in childhood is associated with elevated CRP in adulthood (Juonala et al., 2006); and CRP in childhood is also associated with a variety of cardiovascular (Cook et al., 2000) and atherosclerotic risk factors among youth, such as disturbed endothelial function and intima media thickening, even after adjustment for body mass index (BMI) (Jarvisalo et al., 2002). Although a number of studies have examined the relationship between childhood adversity on inflammation in child or adolescent samples, results have been inconsistent. In a recent systematic review of studies that examined childhood adversity, as measured by either socioeconomic or interpersonal stressors, in relation to inflammation in healthy children and adolescents, the pattern of findings was not clear (Slopen et al., 2011). Some of these studies documented associations between adversity and elevated levels of inflammation (Murasko, 2008; Fuligni et al., 2009a; Howe et al., 2010), while others found no associations (Cook et al., 2000; Gimeno et al., 2008; Miller et al., 2009b), conditional associations (Marin et al., 2007; Danese et al., 2011), or inconsistent associations depending on the measure of adversity (McDade et al., 2005; Dowd et al., 2010).

Some of the inconsistencies across studies may be due to aspects of adversity that prior literature has not been able to consider in detail in relation to inflammation. For example, variation in the type or severity of stressors, or differences in the proximity of stress exposure to assessment of inflammation may account for differences in the impact of 
adversity on inflammatory biomarkers. Furthermore, existing studies have rarely examined childhood adversity and inflammation prospectively in child and adolescent samples, with repeated measures of either the exposures or outcomes. Accordingly, we have limited evidence about whether childhood adversity has persistent effects on inflammation in youth, and whether adversity during early childhood, in contrast to later developmental periods, has a differential impact on later inflammation.

The present study utilized longitudinal data from the Avon Longitudinal Study of Parents and Children (ALSPAC). We used severity-weighted reports of acute adverse events as a measure of childhood adversity. The primary aim was to examine the association between adverse events, measured at seven time points between 1.5 and 8 years of age, and two commonly assessed measures of inflammation, CRP and IL-6. CRP and IL-6 were selected as these inflammatory biomarkers are indicative of systemic inflammation. As it is unclear as to whether CRP per se is causally associated with cardiovascular or other diseases (Casas et al., 2006; Kaptoge et al., 2010), we do not assume that they are on the causal pathway between adversity and coronary heart disease (C Reactive Protein Coronary Heart Disease Genetics Collaboration, 2011) or metabolic syndrome (Timpson et al., 2005). Rather, we consider them as a potential early marker of disease risk, based on prior work demonstrating that elevated levels of CRP are consistently associated with greater atherosclerosis and incident coronary events (Pearson et al., 2003; Danesh et al., 2004). Both IL-6 and CRP were measured at age 10 and CRP was measured again in mid-adolescence (age 15). Repeated assessment of adverse events allowed us to examine the associations of adverse events occurring in specific developmental periods as well as cumulatively with inflammatory processes in childhood and the persistence of those processes into midadolescence.

We hypothesized that adverse events at any time point prior to age 8 would be associated with inflammation at both time points, and the association would be greatest for a cumulative measure that incorporated all assessments of adverse events between 1.5 and 8 years of age. We tested for possible interactions by sex because studies have found sex differences in the relationship between adversity and cardiovascular risk factors in youth (Howe et al., 2010). The second aim was to examine covariates that may explain associations between adverse events and inflammation, including BMI, depression, and cigarette smoking. We selected these potential mediators based on evidence that psychosocial stressors are associated with each of these characteristics and behaviors in youth (Wills et al., 2002; Grant et al., 2004; Gundersen et al., 2011), and that these characteristics are also associated with elevated inflammation (Howren et al., 2009; O'Connor et al., 2009; Dowd et al., 2010).

\section{Methods}

\subsection{Sample}

Participants in this study were drawn from ALSPAC, a population-based prospective longitudinal study of children born to mothers living in Avon county while pregnant (Golding and the ALSPAC Study Team, 2004). ALSPAC was designed to provide insight on how social, biological, and environmental factors interact to influence pregnancy outcomes and child physical and mental health. A total of 14,541 pregnant women with estimated delivery dates between April 1991 and December 1992 agreed to participate, which included roughly $85 \%$ of all eligible participants. The study aimed to follow the 13,988 children who were still alive at 12 months. A series of questionnaires were sent to the parents during the pregnancy and at regular intervals postpartum. Methodological details of the study have been reported previously (Golding et al., 2001; Golding and the ALSPAC Study Team, 2004), and are also described on the study website (www.al-spac.bris.ac.uk). 
The study was approved by the ALSPAC Law and Ethics Committee and Local Research Ethics Committee. Children who dropped out of the study over the course of follow-up were more likely to have behavior disorders and to have mothers with low educational qualifications, financial difficulties, and who smoked (Wolke et al., 2009).

All surviving children whose parents consented to continued participation and with valid contact information were invited to participate in biological assessments. A total of 7725 attended the age 9 clinic (62\% of those invited), and 5253 children attended the age 15 clinic (51\% of those invited). Blood samples were taken from those participants who were willing to participate in the venipuncture protocol (5086 at the age 10 assessment; 3476 at the age 15 assessment). Because active infection may cause elevations in inflammation unrelated to childhood adversity, we excluded individuals who reported an infection in the 7 days prior to blood collection (431 children at the age 10 assessment; 190 adolescents at the age 15 assessment). Children who had at least one measure of CRP or IL-6 collected during assessments at 10 and 15 years of age were eligible for the present study.

Associations between adverse events and inflammation were examined among the 5802 ALSPAC participants without infection in the past week, and with at least one measure of CRP or IL-6 at age $10(N=4655$ and 4647 , respectively $)$, or CRP at age $15(N=3286)$. The exact number of participants in each analysis varies based on the availability of questionnaire data at each assessment of adverse events. This information is provided as a footnote in the tables.

\subsection{Measures}

2.2.1. Inflammatory outcomes-Blood draws at age 10 and 15 were used to assess concentrations of IL-6 (age 10 only) and CRP (age 10 and 15). High sensitivity IL-6 (pg/ $\mathrm{mL}$ ) was measured by enzyme-linked immunosorbent assay (R\&D systems, Abingdon, UK). $\mathrm{CRP}(\mathrm{mg} / \mathrm{L})$ was measured by automated particle-enhanced immunoturbidimetric assay (Roche, UK). Assay coefficients of variation for both outcomes were $<5 \%$. Both CRP and IL-6 were log-transformed for analysis. Following standard practices (Danese et al., 2011), observations that were more than three standard deviations below or above the sample mean (Age 10: IL-6, $N=49$; CRP, $N=43$; Age 15 CRP, $N=40$ ) were winsorized to prevent potential bias due to outliers in linear models (Tukey, 1977; Wilcox and Keselman, 2003).

2.2.2. Adverse life events-When children were $1.5,2.5,3.5,5,6,7$, and 8 years old, mothers were asked whether their children had experienced any of 5 adverse events in the previous 12 to 18 months, and (if it occurred) to rate the impact of the event on the child on a 4-point scale $(0=$ no experience of the event, $4=$ very upset by the event). The same 5 adverse events were included at each time point: (1) taken into some form of foster care; (2) physically hurt by someone; (3) sexually abused; (4) separated from mother; (5) separated from father. A total adverse event score for each time point was calculated by summing the perceived impact score for each event, following a common approach in the literature (Allen et al., 2011). We required that individuals had complete data on all 5 items in order to calculate an adverse events score for a given time point. We also created a cumulative adverse events score by summing the perceived impact scores across the 7 time points (this variable required complete data for all 7 adverse events assessments). We transformed the individual adverse events scores and the cumulative score into $z$-scores to improve normality of the distributions, and increase interpretability of the beta-coefficients. Accordingly, these beta-coefficients refer to the change in the outcome associated with a 1 SD change in the adverse events $z$-score.

We chose to use a measure of perceived impact, rather than a simple count of adverse events because the weighted measure incorporates information about the effect of the event on the 
child, which may reflect severity of the event as well as individual differences in response to the event. Unlike a simple count, the weighted measure does not assume that different events have an equivalent impact (Enoch et al., 2010). For comparison, we replicated our analyses using count score (described in sensitivity analyses, below).

\subsubsection{Potential mediators}

2.2.3.1. BMI: At the time of blood collection, clinic staff measured height using the Harpenden Stadiometer to the last complete millimeter, and unclothed weight to $0.1 \mathrm{~kg}$. BMI was calculated as weight $(\mathrm{kg}) / \mathrm{length}(\mathrm{m})^{2}$.

2.2.3.2. Depression: We utilized depression assessments prior to the blood collection, in order to ensure the temporal ordering required for mediation.

For the age 10 outcomes, we used a depression measure obtained at age 8, assessed by the Development and Well-being Assessment, a validated parent-report structured interview that evaluates the presence of psychiatric diagnoses according to ICD-10 criteria (Goodman et al., 2000). In sensitivity analyses (described below) that required a continuous measure of depressive symptoms, we utilized the emotional symptoms score from the Strengths and Difficulties Questionnaire (Goodman, 2001), the closest available approximation for continuous depressive symptoms available at age 8 .

For the analysis of CRP at age 15, we used a 13-item self-report inventory of depressive symptoms, the Short Moods and Feelings Questionnaire, obtained at age 14 (Angold et al., 1995). This scale correlates highly with the Children's Depression Inventory, the Diagnostic Interview Schedule for Children, and clinical interviews (Wood et al., 1995). A score of 11 or greater was used to create a dichotomous indicator of depression (Angold et al., 2002; Patton et al., 2008). In sensitivity analyses, we utilized the sum of this inventory as a continuous score.

2.2.3.3. Cigarette smoking: Self-reported regular cigarette use at age 15 was assessed at the clinic visit, based on self-report of daily smoking in the past 30 days (yes, no).

2.2.4. Sociodemographic covariates-We included a number of individual and family covariates that are known to be associated with variation in CRP (O'Connor et al., 2009) or that have been used as covariates in similar studies (Caserta et al., 2008; Murasko, 2008; Fuligni et al., 2009b; Copeland et al., 2012), including maternal education, household income, and the child's age, race, and ethnicity. During pregnancy, mothers reported their highest educational attainment (below O-level, O-level only, A-level only, university degree or more, missing), and at birth she reported the child's sex (male, female), and ethnicity (white, non-white). When the child was 8 years, mothers reported weekly household income (<£100, £100-£199, £200-£299, £300-£399, >£400, unknown). At the age 10 and 15 blood collection visits, respondents reported their age (months), and clinic staff recorded whether the respondent was currently taking medication (parent report at age 10, respondents report at age 15; yes, no). Maternal depression, measured by the Edinburgh Postnatal Depression Scale (Cox et al., 1987), was used in sensitivity analyses to test for potential confounding (described below).

2.2.5. Statistical analyses-We used linear regression to estimate associations between the adverse event $z$-scores at each of the 7 time points between 1.5 and 8 years, and the cumulative events $z$-score, and IL-6 and CRP at age 10. Initial models were adjusted for gender, age at blood collection, and ethnicity, and we replicated these models with additional adjustment for other potential confounders including family income at age 8 , 
mother's education at child's birth, and whether or not the child was taking any medication at the time of blood collection. Next, using an identical method, we estimated associations between adverse events $z$-scores at each age and CRP at age 15 . We present the latter models with and without adjustment for age $10 \mathrm{CRP}$. Gender differences in associations were examined by including interaction terms for sex by events $z$-scores.

We then examined the extent to which the observed associations between adverse events and inflammation were mediated by BMI, depression, or regular smoking (age 15 outcome only). We tested the significance of these mediators using a bootstrapping approach that allows for multiple mediators to examined within a single model (Preacher and Hayes, 2008). We obtained indirect effect estimates for each potential mediator, which describes the degree of mediation that occurred (i.e., the proportion of the association between the adverse event score and the inflammatory outcome that is explained by the potential mediator) (Preacher and Hayes, 2008). This approach provides indirect estimates for each potential mediator individually, while other potential mediators are included in the model, and a combined estimate for all potential mediators included in the model. Confidence intervals are provided for all indirect effects; confidence intervals that do not include 0 indicate a significant indirect effect. Mediation models were adjusted for the same covariates noted above.

To examine the robustness of our findings, we performed a number of sensitivity analyses. First, we tested for potential bias in using the perceived impact ratings for the adverse events score, given that both un-weighted and weighted assessments of life events have strengths and limitations (Paykel, 1983) and it is unclear which should be favored in research using parent-report. We repeated our analyses using counts of adverse events, rather than the perceived impact score, to determine if the results were comparable. We also repeated our analyses adjusted for maternal depression levels prior to reported adverse events, given that maternal mood could potentially influence both event impact ratings (Mathews and MacLeod, 2005) and immune activity in children (Caserta et al., 2008).

Second, we repeated our mediation analyses using alternative assessments of the mediators, to examine if our findings were influenced by the measures selected for the primary analyses. We substituted waist circumference for BMI, in order to examine the stability of our results with an alternative measure of adiposity. We also substituted continuous symptoms of depression for the dichotomous indicators, given that some prior studies have examined continuous symptoms as mediators of the relationship between childhood adversity and CRP (Taylor et al., 2006) while other studies have focused on dichotomous indicators of depression (Danese et al., 2007).

Third, we tested depression as an effect modifier, based on studies that have found childhood adversity may only lead to inflammation in the context of major depression (Danese et al., 2008; Danese et al., 2011). Fourth, we repeated our analyses with an interaction term between SES and adverse events to evaluate if the associations differ based on family SES. Specifically, we examined these interactions for maternal education at gestation, and family income at age 8. Fifth, we replicated our analyses using complete cases only, rather than list-wise deletion (which preserved individuals in some models who did not have data for all 7 adverse events scores), in order to evaluate whether the decision to use list-wise deletion impacted our findings. Finally, given the likelihood that adverse experiences at different ages are correlated, we examined if existing significant associations between adverse events at a given time point and the outcomes were independent of adverse events at other time points. All analyses were conducted in SAS v.9.2, and alpha was set at 0.05 . 


\section{Results}

Table 1 describes characteristics of the sample, including means and proportions for the main predictors, outcomes, and covariates. The sample had a similar proportion of males and females, and was predominantly white (95\%), consistent with the general population in Avon county. There was considerable heterogeneity in maternal education and family income. Exposure to each of the adverse events was relatively uncommon. Across the 7 time points, the number of children who experienced each adverse event ranged as follows: taken into care: $3-5$; physically hurt by someone: $122-181$; sexually abused: $1-8$; separated from mother: $125-383$; separated from father: $286-876$. As expected, at age 10 CRP and IL-6 were significantly correlated with each other $(r=0.46, p<.0001)$; and each was also significantly correlated with CRP at age 15 (age 10 CRP: $r=0.32, p<.0001$; age 10 IL-6: $r$ $=0.10, p<.0001)$.

Below, we present results for the combined sample, because there was no evidence for significant gender differences. Models that only adjusted for gender, age, and ethnicity did not differ in any meaningful ways from models that additionally adjusted for family income, mother's education, and medication use; therefore, we only present the fully adjusted models.

\subsection{Adverse events and IL-6 and CRP at age $\mathbf{1 0}$}

Table 2 presents the linear regression of IL- 6 and CRP on adverse events scores measured at each time-point and cumulatively, adjusted for sociodemographic factors and medication use. Adverse events at the first 5 time points (1.5 through 6 years) were not associated with either IL-6 or CRP. The adverse events score at 7 years was associated with elevated IL-6 ( $\beta$ $=0.07, p<.001)$ and $\operatorname{CRP}(\beta=0.06, p=.002)$, and similar associations were observed between the adverse events score at 8 years and both IL- $6(\beta=0.05, p<.001)$ and CRP ( $\beta=$ $0.04, p=.03)$. The cumulative adverse events score was also associated with IL-6 $(\beta=0.04$, $p=.03)$ and CRP $(\beta=0.06, p=.01)$. These beta coefficients reflect the increase in log-CRP or $\log$-IL-6 associated with a $1 \mathrm{SD}$ increase in the adverse events $z$-score.

\subsection{Adverse events and CRP at age $\mathbf{1 5}$}

Table 3 presents associations between adverse events between 1.5 and 8 years of age and CRP at age 15 . The adverse events score at 1.5 years was significantly associated with CRP at 15 years, with higher adversity predicting elevated CRP $(\beta=0.06, p=.001)$. Adverse events at 2.5, 3.5, 4.5, 6, and 7 years were not associated with age $15 \mathrm{CRP}$; however, age 15 CRP was significantly associated with the most proximately assessed adverse events, including events measured at 8 years $(\beta=0.05, p=.02)$ and the cumulative events score ( $\beta$ $=0.05, p=.04)$.

Next, we examined these associations in models that adjusted for CRP at age 10 to determine whether adverse events were associated with change in CRP from late childhood to mid-adolescence. The associations between adverse events at 1.5 years and 8 years and age $15 \mathrm{CRP}$ remained significant in models that adjusted for age $10 \mathrm{CRP}$ (adverse events at 1.5 years: $\beta=0.06, p=.01 ; 8$ years: $\beta=0.06, p=.02)$ whereas the association between the cumulative adverse event score and age $15 \mathrm{CRP}$ was no longer significant $(p=0.18)$. Unexpectedly, adverse events at 6 years was associated with lower CRP at 15, after adjustment for age $10 \mathrm{CRP}(\beta=-0.06, p=.02)$.

\subsection{Potential mediators of inflammation}

3.3.1. Age 10 IL-6 and CRP-For age 10 outcomes we examined BMI and depression as potential mediators. The bottom panel of Table 2 presents the results of mediation analyses 
for the previously reported associations between adverse events and inflammation at age 10 . For IL-6, the mediation models showed that the associations between adverse events reported at 7 and 8 years were maintained even after adjusting for BMI and depression. However, both models revealed a small, but significant indirect effect of adverse events on IL-6 through BMI (adverse events at 7 years (i.e., partial mediation): $\beta=0.01,95 \%$ CI: $0.003-0.02$; adverse events at 8 years: $\beta=0.01,95 \%$ CI: $0.001-0.02)$. In contrast, the association between the cumulative adverse events score and age 10 IL- 6 was mediated by BMI (indirect effect of BMI: $\beta=0.01,95 \%$ CI: $0.002-0.02$ ) to the extent that this association was no longer significant. The indirect effects of depression were not significant in any of the models.

For CRP, the mediation models indicated that all of the previously identified significant associations between adverse events and CRP were mediated by BMI to the extent that the associations between childhood adversity and age 10 were not significant with BMI included in the models. The coefficients for the indirect effects of adverse events on age 10 CRP through BMI were significant for adverse events at 7 years $(\beta=0.03,95 \%$ CI: $0.01-$ $0.05), 8$ years ( $\beta=0.02,95 \%$ CI: $0.003-0.04)$, and the cumulative adverse events score ( $\beta=$ $0.03,95 \%$ CI: 0.01-0.05). Similar to IL-6, the indirect effects of depression were not significant in any of the models. We do not present the total indirect effects for the age 10 CRP or IL-6 mediation models (i.e., the combined indirect effect of BMI and depression), because BMI was the only significant mediator.

3.3.2. Age 15 CRP—For age 15 outcomes, we examined smoking in addition to depression and BMI as potential mediators. The bottom panel of Table 3 presents mediation models for the significant associations between adverse events and CRP at age 15. The association between adverse events reported at 1.5 years and age 15 CRP was not mediated by BMI, depression, or smoking; the direct effect of adverse events was unchanged from the original model, and the indirect effects through the potential mediators, both individually and combined, are not significant (total indirect effect: $\beta=0.002,95 \%$ CI: $-0.01,0.02$ ). Similarly, the association between adverse events reported at 8 years and age 15 CRP was not mediated by BMI, depression, or smoking (total indirect effects: $\beta=0.02,95 \% \mathrm{CI}$ : $-0.0003,0.03)$. In contrast, the association between the cumulative adverse events score and age 15 CRP was mediated by BMI (indirect effect through BMI: $\beta=0.02,95 \%$ CI: 0.01 , 0.04); there were not significant indirect effects of depression or smoking in this model (we do not present the total indirect of BMI, depression, and smoking for this model, because only BMI had a significant indirect effect). As shown in Table 3, these mediation results were similar when age $10 \mathrm{CRP}$ was included as a control covariate.

\subsection{Sensitivity analyses}

We performed several sensitivity analyses to examine the stability of our findings. First, we replicated our models using a count of adverse events, rather than the weighted impact scores (Appendix A1), in order to test for potential bias in using the perceived impact ratings. These analyses revealed a nearly identical pattern of findings, with the exception that the cumulative events score was no longer associated with IL-6 at age 10 and CRP at age 15 . Therefore, the results were largely comparable, although the perceived impact scores were slightly more sensitive for the associations between inflammation and cumulative events. We also tested for potential bias in perceived impact of adverse events resulting from maternal depression. We repeated the original models for which significant associations were observed between adverse events and inflammatory markers with additional adjustment for maternal depression (assessed when the child was 1.5 years, for the model of events at 1.5 years; assessed when the child was 6 years, for models including events at 7 and 8 years); again, the associations were largely unchanged (data not shown). 
Second, we replicated our mediation analyses, using alternative assessments of the mediators, to ensure our findings were not influenced by our choice of measures. When we included waist circumference as a mediator instead of BMI, the results were largely the same. Next, we repeated the mediation analyses using continuous depression symptom scores, rather than diagnostic indicators (for age 10 outcomes, an emotional symptoms score from the SDQ at age 8; for the age 15 outcome, the MFQ symptom score at 13 years); the results were unchanged. Consistent with the dichotomous indicators, the symptom scores did not function as mediators.

Third, we tested whether depression modified the association between adverse events and inflammation. We did not find evidence for depression as an effect modifier in this sample (i.e., all $p$-values for interactions between depression and adverse events $z$-scores $>0.05$ ). Fourth, we did not find evidence that SES moderates the association between adversity and inflammation (considering either maternal education at gestation, or household income at age 8). Fifth, we repeated our analyses using only complete cases for the age 10 and age 15 outcomes (Appendix A2). There was one noteworthy difference: in the complete case analysis (which required that children had adverse events reported at all seven time points), the adverse events score at 1.5 years was not associated with CRP at age $15(\beta=0.04, p=$. $10)$. Given that high-risk families were least likely to return the questionnaires consistently (Wolke et al., 2009), complete-case analysis excludes children with the highest adverse events scores at 1.5 years. Therefore, we presented list-wise deletion analyses in order to maximize available data and minimize potential bias. Finally, we examined if the existing significant associations were independent of adverse events scores at other time points. In regression models that included all 7 adverse events scores together, the results were largely unchanged.

\section{Discussion}

The present study used data from a population-based prospective cohort of healthy children to examine the associations between adverse events collected from age 1.5 to 8 years and inflammation at ages 10 and 15 . Adverse events in middle childhood (occurring between ages 6 to 8 ), as well as adverse events cumulated from age 1.5 through age 8 , were associated with higher levels of IL-6 and CRP at age 10. In contrast, adverse events experienced earlier in childhood (i.e., reports between 1.5 and 6 years) were not associated with age 10 inflammation. Adverse events in early childhood (age 1.5), middle childhood (age 8), and the cumulative events score predicted increased levels of CRP at the age 15 assessment. Importantly, these associations persisted after adjustment for age $10 \mathrm{CRP}$, suggesting that adverse events in early and middle childhood were associated with a greater increase in level of CRP from age 10 to 15 . Some of these associations were mediated by BMI, whereas we found no evidence that depression or smoking mediated the association between adverse events and inflammation. A key finding from this analysis was that the associations between the cumulative adverse events score and inflammation at ages 10 and 15 were not of greater magnitude than the significant associations for measures that were most proximate to the outcomes; contrary to our hypothesis that the associations would be greatest for the cumulative measure of adverse events, our findings suggest that effects may not be cumulative per se.

Our results suggest inflammation as an early mechanism by which adverse experiences in childhood become biologically embedded and adversely impact health over the life course. Our study is unique in providing insight on the temporal relationship between adverse events in early and middle childhood, subsequent inflammation, and the persistence of inflammation. These results correspond with prior research indicating elevated levels of inflammation among children and adolescents (Murasko, 2008; Dixon et al., 2009; Fuligni et 
al., 2009a; Howe et al., 2010) and adults (Taylor et al., 2006; Danese et al., 2007; Pollitt et al., 2007; Taylor et al., 2011) who experienced adversity in early life. These findings may also explain some of the inconsistencies across earlier studies. Specifically, timing of exposure to adverse events appears to matter - with adverse events in early childhood and relatively proximate to the measure of inflammation most strongly associated with inflammation. Thus, if prior studies had varying lengths of time between measurement of adverse events and inflammation, these associations may have been more difficult to detect. Our findings are inconsistent with some other studies that have examined stressful events to inflammation, such as a prospective study that did not find an association between interpersonal stressors and circulating levels of CRP or IL-6 in a sample of female adolescents (mean age, 17 years) (Miller et al., 2009b). The disparate findings may be due to differences in the age of our samples, sample sizes, timing and type of experiences assessed, or time to follow-up.

There are at least two potential explanations for the observed associations between age 10 inflammation and adverse events reported at 7 and 8 years, but not earlier reports of adverse events. It is possible that adverse events occurring later in childhood have a more pronounced influence on inflammation relative to earlier in childhood; or this pattern may be a consequence of the fact that the age 7 and 8 reports were provided in the closest proximity to inflammatory assessments and inflammatory consequences of earlier childhood adversity are relatively immediate and short-lived. It may be that earlier adverse events had a significant impact on inflammatory markers, but these effects dissipated or were reversed prior to the age 10 assessments. Research suggests that physical and emotional trauma can cause relatively short-lived elevations IL-6 in children (Pervanidou et al., 2008), suggesting that both alternatives are plausible.

At age 15, both recent (age 8) and early-life (age 1.5) adverse events were associated with elevated CRP. A potential explanation for this finding is that adverse events early in life cultivated a pro-inflammatory phenotype characterized by alterations in signaling molecules that affect the regulation of inflammation, and these alterations were not evident in circulating biomarkers of inflammatory activity until a later stage of physiological development (i.e., in our study, mid-adolescence). For example, Miller and colleagues (Miller and Chen, 2007; Miller and Chen, 2010) found that harsh family climate predicted IL-6 response to lipopolysaccharide exposure (a bacterial stimuli) but did not predict circulating levels of IL-6 in normal conditions among adolescent females. Thus, in our study, it is possible that the influence of early adversity (e.g., at 1.5 years) is not evident in basal levels of inflammation in healthy children at age 10; however, these children may have an amplified inflammatory response to exogenous stimuli, such that over time repeated excessive inflammatory responses leads to chronic inflammation (e.g., detectable at age 15). In order to gain insight on this hypothesized explanation for our findings, future birth cohorts should include multiple measurements of inflammation and pro-inflammatory signaling molecules, along with multiple measures of psychosocial stress and the social environment, from birth onwards.

Results from the mediation analyses are only partially consistent with existing literature. We found evidence of mediation through BMI for some of the associations, which corresponds with prior studies of childhood adversity and inflammation in children (Howe et al., 2010) and adults (Taylor et al., 2006). For example, in a study that examined maternal education in relation to cardiovascular disease risk factors in ALSPAC, almost the entire association between maternal education and CRP was mediated by adiposity; and for IL-6, there was also evidence of mediation through adiposity, but to a lesser extent (Howe et al., 2010). In our study, BMI attenuated to varying degrees the associations between cumulative adverse events and proximate events and inflammation. Our study did not support smoking or 
depression as mediators (i.e., the indirect effects were not significant), which is inconsistent with some prior research in adult samples which identify smoking (Pollitt et al., 2007) and psychological functioning (Taylor et al., 2004; Taylor et al., 2006) as mediators. It is possible that smoking did not function as a significant mediator, given the low prevalence of smoking at age 15 in this sample and possibly the limited duration of smoking. In the age 10 mediation analyses of childhood adversity reported at 8 years, somewhat surprisingly depression had a significant inverse association with CRP, notably, this association is not significant when depression is the only mediator in the model (i.e., if BMI is not included). In contrast, in the models of age $15 \mathrm{CRP}$, we observe a positive association between depression and greater CRP, which is consistent with prior research using adult samples (Taylor et al., 2004, 2006). Although it is challenging to identify comparable mediation analyses in child and adolescent samples, several studies that examined childhood adversity and inflammation in children (Wolf et al., 2008; Fuligni et al., 2009a) and adults (Danese et al., 2007) found that associations persist even after adjustment for psychological functioning and smoking, consistent with our findings.

There are several limitations of the present study. First, our study is limited by incomplete data and some loss-to-follow up. Similar to most prospective cohorts, continued and consistent participation was socially patterned, and individuals from more disadvantaged circumstances were less likely to participate continuously. In line with this, individuals who did not participate in the age 10 or age 15 blood assessments had greater cumulative adversity scores relative to participants (e.g., the mean cumulative adversity $z$-score assessment for non-participants at age 10 is greater than the mean score for participants (mean $z$-score $=0.02, \mathrm{SD}=1.05$ vs. mean $z$-score $=-0.02, \mathrm{SD}=0.96$, respectively $)$ ). We controlled for variables associated with attrition (e.g., maternal education, income) which may help to minimize bias (Kleinbaum et al., 1982). Nonetheless, this non-differential missingness suggests that our data cannot be generalized to the original populationrepresentative cohort, and may have resulted in attenuation of the observed associations. Second, although it has more measures than most, our study is limited by only two assessments of inflammation. It is not possible to make conclusions about the lack of associations between the earlier measures of adverse events and inflammation, because short-term elevations may have occurred that dissipated prior to age 10. Third, given the observational design, we are not able to conclude definitively that the observed associations are causal; however, these associations are consistent with experimental research demonstrating that fluctuations in circulating markers of inflammation occur in response to acute psychological stressors (Steptoe et al., 2007). Furthermore, our evaluation of age 15 CRP examined within-person change, which strengthens causal inference within observational studies (Rutter, 2007). Fourth, we had imperfect control for household income at age 8 , given that a substantial proportion of caregivers did not provide this information. Several sensitivity analyses (e.g., omitting individuals with missing income information; models not including household income) revealed that our findings are robust to this limitation. Finally, it is likely that adverse events were under-reported as this information was provided by care-givers. Any under-reporting would have biased our findings towards the null.

Despites these limitations, our study is a valuable addition to the literature because it is among the first prospective studies of children documenting that exposure to adverse events prior to age 8 increased inflammation at age 10 , and that effects of adverse events on inflammation are sustained into adolescence. These findings provide evidence for a biological mechanism by which early experiences shape long-term health, and highlight the importance of developing interventions that can minimize both the occurrence and consequences of childhood adversity on physiological processes during sensitive periods of development. 
Our study has implications for both research and practice. Research is needed to gain insight on the long-term clinical significance of elevated inflammation during childhood and adolescence. For example, for each $1 \mathrm{SD}$ increase in the adverse events score at age 7, age 10 CRP increases by $7.3 \%$, and IL-6 increases by $6.2 \%$. At present, it is unclear whether these increases reflect dysregulated processes that could precede or mark increased risk for later chronic disease development. Further studies of children with earlier assessments of inflammation are necessary in order to more precisely elucidate mechanisms that connect childhood adversity to later disease vulnerability, and to identify potential opportunities to slow the progression of or minimize biological dysregulation. In addition, future research is needed to explore our finding that adverse events in closer proximity to the assessments of inflammation were most consistently associated with inflammation, and to determine if this finding holds in models that adjust for inflammation prior to the adverse event. There is also a need for more nuanced examinations of the relationship between psychological and emotional functioning and inflammation in children and adolescents. Contrary to existing research with adults (Taylor et al., 2004, 2006), depression was not a significant mediator in our analysis. In light of recent research showing that depression is prospectively associated with elevated levels of CRP over the course of adolescence (Copeland et al., 2012), additional attention to this issue in child and adolescent samples is warranted. Finally, it may be useful for future research to examine these associations in low- and middle-income countries, as research suggests that social exposures may relate to inflammation differently based on environmental and sociocultural conditions (Nazmi et al., 2010).

From a practice perspective, it may be valuable for researchers to incorporate assessments of inflammation into prevention-oriented intervention studies for high-risk children and adolescents. This will provide insight on whether it is possible to reverse or minimize dysregulated inflammatory processes, and could inform interventions. In the future, practitioners may be able to utilize biomarkers of inflammation to identify children and adolescents at high risk for long-term health consequences, or to evaluate the effectiveness of social interventions to prevent future chronic disease risk among high-risk children.

\section{Acknowledgments}

\section{Role of funding source}

The study sponsors were not involved in analysis, interpretation of data, writing of this report, or decision to submit this paper for publication.

We are extremely grateful to all the families who took part in this study, the midwives for their help in recruiting them, and the whole ALSPAC team, which includes interviewers, computer and laboratory technicians, clerical workers, research scientists, volunteers, managers, receptionists and nurses. The UK Medical Research Council (Grant ref.: 74882) the Wellcome Trust (Grant ref.: 076467) and the University of Bristol provide core support for ALSPAC. This publication is the work of the authors and will serve as guarantors for the contents of this paper. This research was specifically funded by postdoctoral fellowship to the first author from the Robert Wood Johnson Foundation to support the Early Childhood Innovation Project. The authors would also like to thank Dr. Kate Northstone for her data assistance.

\section{References}

Allen J, Rapee R, Sandberg S. Assessment of maternally reported life events in children and adolescents: a comparison of interview and checklist methods. J Psychopathol Behav. 2011:1-12.

Angold A, Costello EJ, Messer SC, Pickles A, Winder F, Silver D. Development of a short questionnaire for use in epidemiological studies of depression in children and adolescents. Int $\mathbf{J}$ Methods Psychiatr Res. 1995; 5:237-249.

Angold A, Erkanli A, Silberg J, Eaves L, Costello EJ. Depression scale scores in 8-17-year-olds: effects of age and gender. J Child Psychol Psyc. 2002; 43:1052-1063. 
C Reactive Protein Coronary Heart Disease Genetics Collaboration. Association between C reactive protein and coronary heart disease: mendelian randomisation analysis based on individual participant data. BMJ. 2011:342.

Casas JP, Shah T, Cooper J, Hawe E, McMahon AD, Gaffney D, Packard CJ, O'Reilly DS, JuhanVague I, Yudkin JS, Tremoli E, Margaglione M, Di Minno G, Hamsten A, Kooistra T, Stephens JW, Hurel SJ, Livingstone S, Colhoun HM, Miller GJ, Bautista LE, Meade T, Sattar N, Humphries $\mathrm{SE}$, Hingorani AD. Insight into the nature of the CRP-coronary event association using Mendelian randomization. Int J Epidemiol. 2006; 35:922-931. [PubMed: 16565153]

Caserta MT, O’Connor TG, Wyman PA, Wang H, Moynihan J, Cross W, Tu X, Jin X. The associations between psychosocial stress and the frequency of illness, and innate and adaptive immune function in children. Brain Behav Immun. 2008; 22:933-940. [PubMed: 18308510]

Chapman DP, Whitfield CL, Felitti VJ, Dube SR, Edwards VJ, Anda RF. Adverse childhood experiences and the risk of depressive disorders in adulthood. J Affect Disorders. 2004; 82:217225. [PubMed: 15488250]

Cook DG, Mendall MA, Whincup PH, Carey IM, Ballam L, Morris JE, Miller GJ, Strachan DP. Creactive protein concentration in children: relationship to adiposity and other cardiovascular risk factors. Atherosclerosis. 2000; 149:139-150. [PubMed: 10704625]

Copeland WE, Shanahan L, Worthman C, Angold A, Costello EJ. Cumulative depression episodes predict later C-reactive protein levels: a prospective analysis. Biol Psychiatr. 2012; 71:15-21.

Cox JL, Holden JM, Sagovsky R. Detection of postnatal depression development of the 10-item Edinburgh Postnatal Depression Scale. Br J Psychiatr. 1987; 150:782-786.

Danese A, Caspi A, Williams B, Ambler A, Sugden K, Mika J, Werts H, Freeman J, Pariante CM, Moffitt TE, Arseneault L. Biological embedding of stress through inflammation processes in childhood. Mol Psychiatr. 2011; 16:244-246.

Danese A, Moffitt TE, Harrington H, Milne BJ, Polanczyk G, Pariante CM, Poulton R, Caspi A. Adverse Childhood Experiences and Adult Risk Factors for Age-Related Disease Depression Inflammation, and Clustering of Metabolic Risk Markers. Arch Pediat Adol Med. 2009; 163:1135-1143.

Danese A, Moffitt TE, Pariante CM, Ambler A, Poulton R, Caspi A. Elevated inflammation levels in depressed adults with a history of childhood maltreatment. Arch Gen Psychiatry. 2008; 65:409415. [PubMed: 18391129]

Danese A, Pariante CM, Caspi A, Taylor A, Poulton R. Childhood maltreatment predicts adult inflammation in a life-course study. Proc Natl Acad Sci USA. 2007; 104:1319-1324. [PubMed: 17229839]

Danesh J, Wheeler JG, Hirschfield GM, Eda S, Eiriksdottir G, Rumley A, Lowe GDO, Pepys MB, Gudnason V. C-reactive protein and other circulating markers of inflammation in the prediction of coronary heart disease. N Engl J Med. 2004; 350:1387-1397. [PubMed: 15070788]

Dixon D, Meng H, Goldberg R, Schneiderman N, Delamater A. Stress and body mass index each contributes independently to tumor necrosis factor-[alpha] production in prepubescent Latino Children. J Pediatr Nurs. 2009; 24:378-388. [PubMed: 19782896]

Dong M, Giles WH, Felitti VJ, Dube SR, Williams JE, Chapman DP, Anda RF. Insights into causal pathways for ischemic heart disease: adverse childhood experiences study. Circulation. 2004; 110:1761-1766. [PubMed: 15381652]

Dowd JB, Zajacova A, Aiello AE. Predictors of inflammation in U.S. children aged 3-16 years. Am J Prev Med. 2010; 39:314-320. [PubMed: 20837281]

Dube SR, Fairweather D, Pearson WS, Felitti VJ, Anda RF, Croft JB. Cumulative childhood stress and autoimmune diseases in adults. Psychosom Med. 2009; 71:243-250. [PubMed: 19188532]

Enoch MA, Steer CD, Newman TK, Gibson N, Goldman D. Early life stress, MAOA, and geneenvironment interactions predict behavioral disinhibition in children. Genes Brain Behav. 2010; 9:65-74. [PubMed: 19804559]

Felitti VJ, Anda RF, Nordenberg D, Williamson DF, Spitz AM, Edwards V, Koss MP, Marks JS. Relationship of childhood abuse and household dysfunction to many of the leading causes of death in adults The Adverse Childhood Experiences (ACE) Study. Am J Prev Med. 1998; 14:245-258. [PubMed: 9635069] 
Fuligni AJ, Telzer EH, Bower J, Cole SW, Kiang L, Irwin MR. A preliminary study of daily interpersonal stress and C-reactive protein levels among adolescents from Latin American and European backgrounds. Psychosom Med. 2009a; 71:329-333. [PubMed: 19196810]

Fuligni AJ, Telzer EH, Bower J, Irwin MR, Kiang L, Cole SW. Daily family assistance and inflammation among adolescents from Latin American and European backgrounds. Brain Behav Immun. 2009b; 23:803-809. [PubMed: 19275931]

Galobardes B, Smith GD, Lynch JW. Systematic review of the influence of childhood socioeconomic circumstances on risk for cardiovascular disease in adulthood. Ann Epidemiol. 2006; 16:91-104. [PubMed: 16257232]

Gimeno D, Ferrie JE, Elovainio M, Pulkki-Raback L, Keltikangas-Jarvinen L, Eklund C, Hurme M, Lehtimaki T, Marniemi J, Viikari JSA, Raitakari OT, Kivimaki M. When do social inequalities in C-reactive protein start? A life course perspective from conception to adulthood in the Cardiovascular Risk in Young Finns Study. Int J Epidemiol. 2008; 37:290-298. [PubMed: 18056120]

Golding J, Pembrey M, Jones R. the Alspac Study Team. ALSPAC-The Avon Longitudinal Study of Parents and Children. Paediatri Perinatal Epidemiol. 2001; 15:74-87.

Golding J. the ALSPAC Study Team. The Avon Longitudinal Study of Parents and Children (ALSPAC)-study design and collaborative opportunities. Eur J Endocrinol. 2004; 151:U119U123. [PubMed: 15554896]

Goodman R. Psychometric properties of the strengths and difficulties questionnaire. J Am Acad Child Adol Psyc. 2001; 40:1337-1345.

Goodman R, Ford T, Richards H, Gatward R, Meltzer H. The development and well-being assessment: description and initial validation of an integrated assessment of child and adolescent psychopathology. J Child Psychol Psyc. 2000; 41:645-655.

Grant KE, Compas BE, Thurm AE, McMahon SD, Gipson PY. Stressors and child and adolescent psychopathology: measurement issues and prospective effects. J Clin Child Adolesc Psychol. 2004; 33:412-425. [PubMed: 15136206]

Gundersen C, Mahatmya D, Garasky S, Lohman B. Linking psychosocial stressors and childhood obesity. Obes Rev. 2011; 12:e54-e63. [PubMed: 21054757]

Howe LD, Galobardes B, Sattar N, Hingorani AD, Deanfield J, Ness AR, Davey-Smith G, Lawlor DA. Are there socioeconomic inequalities in cardiovascular risk factors in childhood, and are they mediated by adiposity? Findings from a prospective cohort study. Int J Obesity. 2010; 34:1149_ 1159.

Howren MB, Lamkin DM, Suls J. Associations of depression with C-reactive protein IL-1, and IL-6: a meta-analysis. Psychosom Med. 2009; 71:171-186. [PubMed: 19188531]

Jarvisalo MJ, Harmoinen A, Hakanen M, Paakunainen U, Viikari J, Hartiala J, Lehtimaki T, Simell O, Raitakari OT. Elevated serum C-reactive protein levels and early arterial changes in healthy children. Arterioscl Throm Vas. 2002; 22:1323-1328.

Juonala M, Viikari JSA, Ronnemaa T, Taittonen L, Marniemi J, Raitakari OT. Childhood C-reactive protein in predicting CRP and carotid intima-media thickness in adulthood - The Cardiovascular Risk in Young Finns Study. Arterioscl Throm Vas. 2006; 26:1883-1888.

Kaptoge S, Di Angelantonio E, Lowe G, Pepys MB, Thompson SG, Collins R, Danesh J. C-reactive protein concentration and risk of coronary heart disease, stroke, and mortality: an individual participant meta-analysis. Lancet. 2010; 375:132-140. [PubMed: 20031199]

Kessler RC, McLaughlin KA, Green JG, Gruber MJ, Sampson NA, Zaslavsky AM, Aguilar-Gaxiola S, Alhamzawi AO, Alonso J, Angermeyer M, Benjet C, Bromet E, Chatterji S, de Girolamo G, Demyttenaere K, Fayyad J, Florescu S, Gal G, Gureje O, Haro JM, Hu CY, Karam EG, Kawakami N, Lee S, Lepine JP, Ormel J, Posada-Villa J, Sagar R, Tsang A, Ustun TB, Vassilev S, Viana MC, Williams DR. Childhood adversities and adult psychopathology in the WHO World Mental Health Surveys. Br J Psychiatr. 2010; 197:378-385.

Kleinbaum, DG.; Kupper, LL.; Morgenstern, H. Epidemiologic Research: Principles and Quantitative Methods. John Wiley and Sons; Belmont, CA: 1982. 
Marin TJ, Martin TM, Blackwell E, Stetler C, Miller GE. Differentiating the impact of episodic and chronic stressors on hypothalamic-pituitary-adrenocortical axis regulation in young women. Health Psychol. 2007; 26:447-455. [PubMed: 17605564]

Mathews A, MacLeod C. Cognitive vulnerability to emotional disorders. Annu Rev Clin Psychol. 2005; 1:167-195. [PubMed: 17716086]

McDade TW, Leonard WR, Burhop J, Reyes-Garcia V, Vadez V, Huanca T, Godoy RA. Predictors of C-reactive protein in Tsimane' 2 to 15 year-olds in lowland Bolivia. Am J Phys Anthropol. 2005; 128:906-913. [PubMed: 16118783]

Miller G, Chen E. Unfavorable socioeconomic conditions in early life presage expression of proinflammatory phenotype in adolescence. Psychosom Med. 2007; 69:402-409. [PubMed: 17556642]

Miller G, Chen E, Cole SW. Health psychology: developing biologically plausible models linking the social world and physical health. Annu Rev Psychol. 2009a; 60:501-524. [PubMed: 19035829]

Miller GE, Chen E. Harsh family climate in early life presages the emergence of a proinflammatory phenotype in adolescence. Psychol Sci. 2010; 21:848-856. [PubMed: 20431047]

Miller GE, Rohleder N, Cole SW. Chronic interpersonal stress predicts activation of pro- and antiinflammatory signaling pathways 6 months later. Psychosom Med. 2009b; 71:57-62. [PubMed: 19073750]

Murasko JE. Male-female differences in the association between socioeconomic status and atherosclerotic risk in adolescents. Soc Sci Med. 2008; 67:1889-1897. [PubMed: 18926611]

Nazmi A, Oliveira IO, Horta BL, Gigante DP, Victora CG. Lifecourse socioeconomic trajectories and C-reactive protein levels in young adults: Findings from a Brazilian birth cohort. Soc Sci Med. 2010; 70:1229-1236. [PubMed: 20137842]

O'Connor MF, Bower JE, Cho HJ, Creswell JD, Dimitrov S, Hamby ME, Hoyt MA, Martin JL, Robles TF, Sloan EK, Thomas KS, Irwin MR. To assess, to control, to exclude: effects of biobehavioral factors on circulating inflammatory markers. Brain Behav Immun. 2009; 23:887897. [PubMed: 19389469]

Patton GC, Olsson C, Bond L, Toumbourou JW, Carlin JB, Hemphill SA, Catalano RF. Predicting female depression across puberty: a two-nation longitudinal study. J Am Acad Child Adolesc Psychiatr. 2008; 47:1424-1432.

Paykel ES. Methodological aspects of life events research. J Psychosom Res. 1983; 27:341-352. [PubMed: 6668560]

Pearson TA, Mensah GA, Alexander RW, Anderson JL, Cannon RO, Criqui M, Fadl YY, Fortmann SP, Hong Y, Myers GL, Rifai N, Smith SC, Taubert K, Tracy RP, Vinicor F. Markers of inflammation and cardiovascular disease application to clinical and public health practice - a statement for healthcare professionals from the centers for disease control and prevention and the American Heart Association. Circulation. 2003; 107:499-511. [PubMed: 12551878]

Pervanidou P, Margeli A, Lazaropoulou C, Papassotiriou I, Chrousos GP. The immediate and longterm impact of physical and/or emotional stress from motor vehicle accidents on circulating stress hormones and adipo-cytokines in children and adolescents. Stress: Int J Biol Stress. 2008; 11:438447.

Pollitt RA, Kaufman JS, Rose KM, Diez-Roux AV, Zeng D, Heiss G. Early-life and adult socioeconomic status and inflammatory risk markers in adulthood. Eur J Epidemiol. 2007; 22:5566. [PubMed: 17225957]

Preacher K, Hayes A. Asymptotic and resampling strategies for assessing and comparing indirect effects in multiple mediator models. Behav Res Meth. 2008; 40:879-891.

Rutter M. Proceeding from observed correlation to causal inference: the use of natural experiments. Perspect Psychol Sci. 2007; 2:377-395.

Shonkoff JP, Boyce WT, McEwen BS. Neuroscience, molecular biology, and the childhood roots of health disparities: building a new framework for health promotion and disease prevention. JAMA. 2009; 301:2252-2259. [PubMed: 19491187]

Slopen N, Kubzansky LD, Koenen KC. Childhood adversity and inflammatory and immune biomarkers associated with cardiovascular risk in youth: a systematic review. Brain Behav Immun. 2011; 26:239-250. [PubMed: 22138616] 
Steptoe A, Hamer M, Chida Y. The effects of acute psychological stress on circulating inflammatory factors in humans: a review and meta-analysis. Brain Behav Immun. 2007; 21:901-912. [PubMed: 17475444]

Taylor SE, Lehman BJ, Kiefe CI, Seeman TE. Relationship of early life stress and psychological functioning to adult C-reactive protein in the coronary artery risk development in young adults study. Biol Psychiatr. 2006; 60:819-824.

Taylor SE, Lerner JS, Sage RM, Lehman BJ, Seeman TE. Early environment, emotions, responses to stress, and health. J Pers. 2004; 72:1365-1393. [PubMed: 15509286]

Taylor SE, Way BM, Seeman TE. Early adversity and adult health outcomes. Dev Psychopathol. 2011; 23:939-954. [PubMed: 21756443]

Thomas C, Hyppönen E, Power C. Obesity and type 2 diabetes risk in midadult life: the role of childhood adversity. Pediatrics. 2008; 121:e1240-e1249. [PubMed: 18450866]

Timpson NJ, Lawlor DA, Harbord RM, Gaunt TR, Day INM, Palmer LJ, Hattersley AT, Ebrahim S, Lowe GDO, Rumley A, Smith GD. C-reactive protein and its role in metabolic syndrome: mendelian randomisation study. Lancet. 2005; 366:1954-1959. [PubMed: 16325697]

Tukey, JW. Exploratory Data Analysis. Addison-Wesley; Reading, MA: 1977.

Von Korff M, Alonso J, Ormel J, Angermeyer M, Bruffaerts R, Fleiz C, de Girolamo G, Kessler RC, Kovess-Masfety V, Posada-Villa J, Scott KM, Uda H. Childhood psychosocial stressors and adult onset arthritis: Broad spectrum risk factors and allostatic load. Pain. 2009; 143:76-83. [PubMed: 19251363]

Wilcox RR, Keselman HJ. Modern robust data analysis methods: measures of central tendency. Psychol Methods. 2003; 8:254-274. [PubMed: 14596490]

Wills TA, Sandy JM, Yaeger AM. Stress and smoking in adolescence: a test of directional hypotheses. Health Psychol. 2002; 21:122-130. [PubMed: 11950102]

Wolf JM, Miller GE, Chen E. Parent psychological states predict changes in inflammatory markers in children with asthma and healthy children. Brain Behav Immun. 2008; 22:433-441. [PubMed: 18068332]

Wolke D, Waylen A, Samara M, Steer C, Goodman R, Ford T, Lamberts K. Selective drop-out in longitudinal studies and non-biased prediction of behaviour disorders. Br J Psychiatr. 2009; 195:249-256.

Wood A, Kroll L, Moore A, Harrington R. Properties of the Mood and Feelings Questionnaire in adolescent psychiatric outpatients-a research note. J Child Psychol Psyc. 1995; 36:327-334.

\section{Appendix A}

Tables A1 and A2. 
Table 1

Characteristics of the sample. ${ }^{a}$

\begin{tabular}{|c|c|c|}
\hline & $N$ & $\%$, Median (SE), or Mean (SD) \\
\hline \multicolumn{3}{|l|}{ Outcomes (median, SE) } \\
\hline Age 10 IL-6 (pg/mL) & 4647 & $0.78(0.02)$ \\
\hline Age 10 CRP (mg/L) & 4655 & $0.20(0.03)$ \\
\hline Age 15 CRP (mg/L) & 3286 & $0.38(0.05)$ \\
\hline \multicolumn{3}{|l|}{ Adverse Events Score (mean, SD) } \\
\hline 1.5 years & 5083 & $0.40(0.97)$ \\
\hline 2.5 years & 4892 & $0.49(1.08)$ \\
\hline 3.5 years & 4913 & $0.46(1.05)$ \\
\hline 4.5 years & 4770 & $0.40(1.06)$ \\
\hline 6 years & 4598 & $0.34(1.02)$ \\
\hline 7 years & 4581 & $0.27(0.95)$ \\
\hline 8 years & 4695 & $0.29(1.00)$ \\
\hline Sum score ( 18 months to 8 years) & 3545 & $2.47(3.52)$ \\
\hline \multicolumn{3}{|l|}{ Gender (\%) } \\
\hline Male & 2910 & 50.16 \\
\hline Female & 2892 & 49.84 \\
\hline \multicolumn{3}{|l|}{ Ethnicity (\%) } \\
\hline Non-White/missing & 308 & 5.31 \\
\hline White & 5494 & 94.69 \\
\hline \multicolumn{3}{|l|}{ Maternal education at birth ${ }^{b}(\%)$} \\
\hline Missing & 499 & 8.6 \\
\hline Below O-level & 1118 & 19.27 \\
\hline O-level only & 1858 & 32.02 \\
\hline A-level & 1444 & 24.89 \\
\hline University degree+ & 883 & 15.22 \\
\hline \multicolumn{3}{|l|}{ Weekly household income (age 8) (\%) } \\
\hline Unknown & 1597 & 27.52 \\
\hline$<£ 100$ & 73 & 1.26 \\
\hline$£ 100-£ 199$ & 322 & 5.55 \\
\hline$£ 200-£ 299$ & 660 & 11.38 \\
\hline$£ 300-£ 399$ & 876 & 15.1 \\
\hline$>£ 400$ & 2274 & 39.19 \\
\hline \multicolumn{3}{|l|}{ Body Mass Index (mean, SD) } \\
\hline Age 10 & 5421 & $17.64(2.81)$ \\
\hline Age 15 & 4186 & $21.42(3.50)$ \\
\hline \multicolumn{3}{|l|}{$\operatorname{Depression}^{c}(\%)$} \\
\hline Age 8 (DAWBA) & 4626 & 0.41 \\
\hline Age 14 (MQF-SF) & 4303 & 11.18 \\
\hline Daily cigarette use (Age 15) (\%) & 270 & 4.65 \\
\hline
\end{tabular}

Psychoneuroendocrinology. Author manuscript; available in PMC 2014 February 01. 
${ }^{a}$ Descriptive statistics for respondents with valid blood data for CRP or IL-6 at Age $10(N=5802)$.

$b_{\text {O-level }}=$ ordinary level; exams taken at age 15-16, the completion of legally required school attendance, equivalent to today's General Certificate of Secondary Education. A-level $=$ advanced level.

${ }^{c}$ DAWBA $=$ Development and Wellbeing Assessment; MFQ-SF = Moods and Feelings Questionnaire, Short-Form. SD = standard deviation; SE $=$ standard error. 


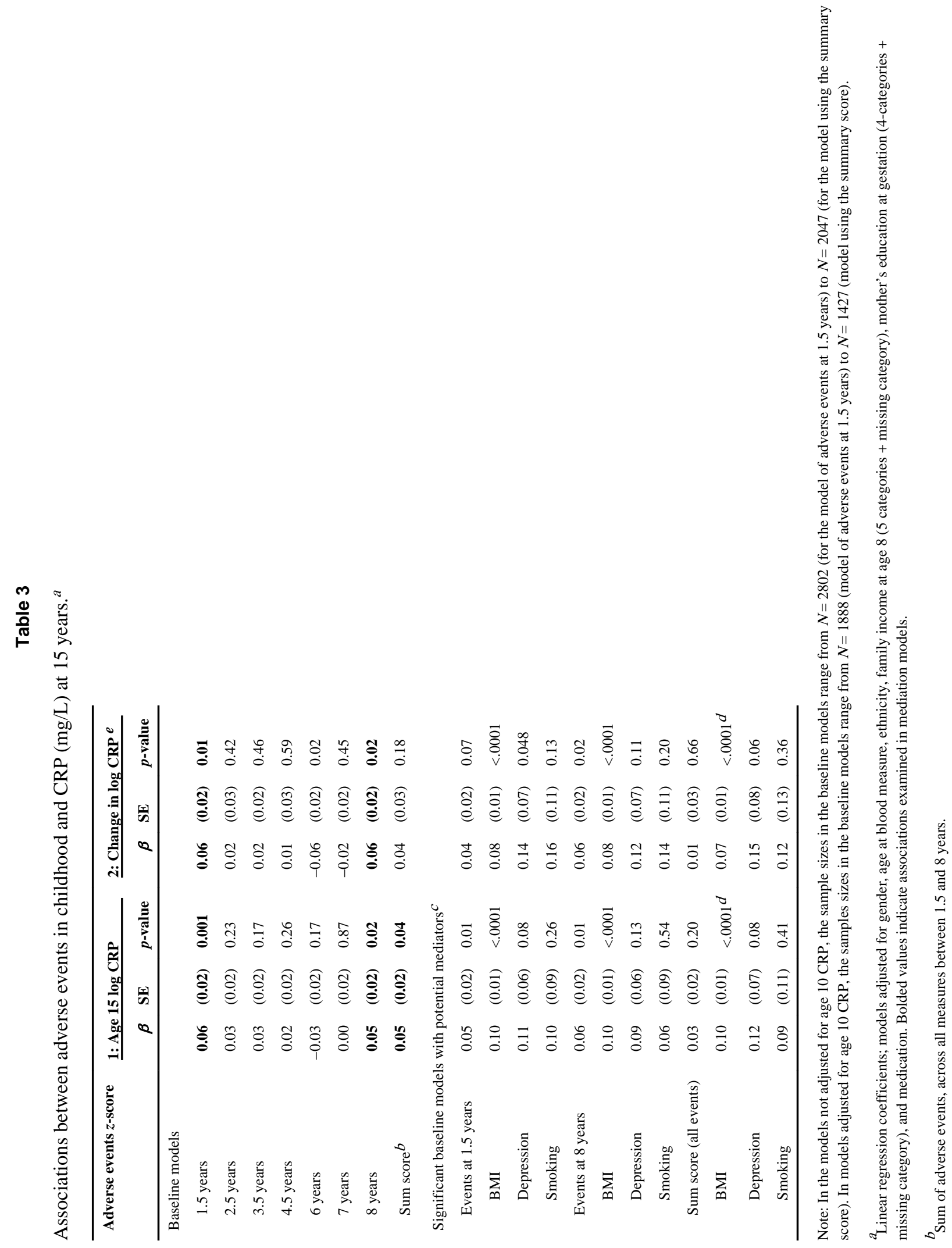




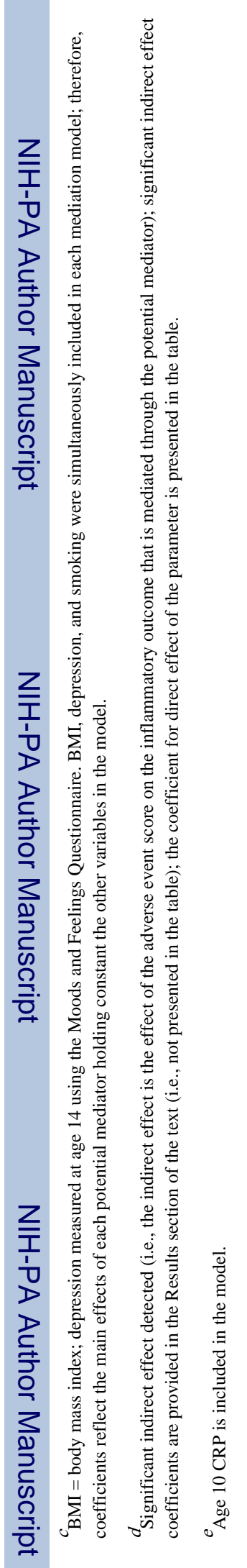




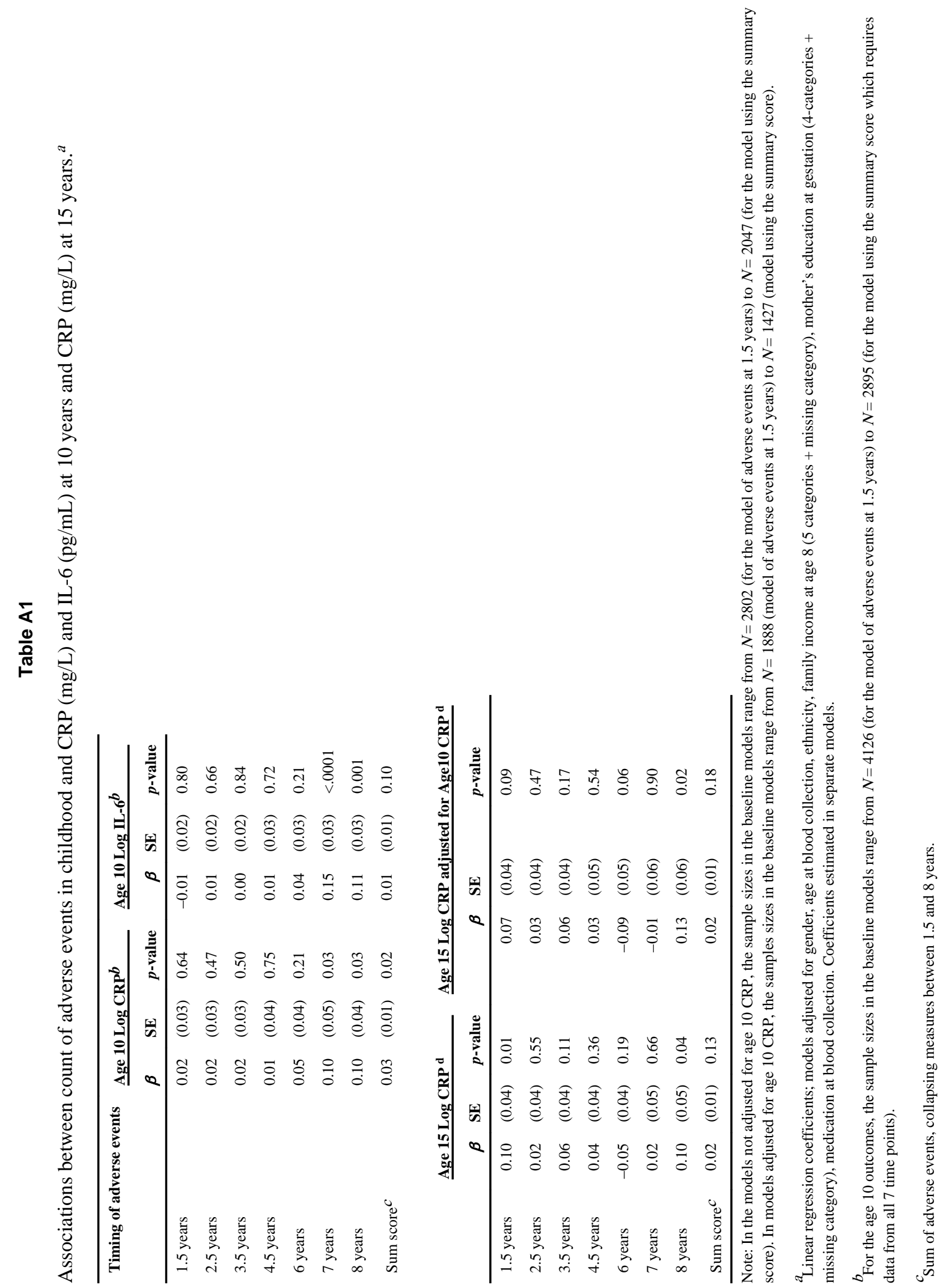




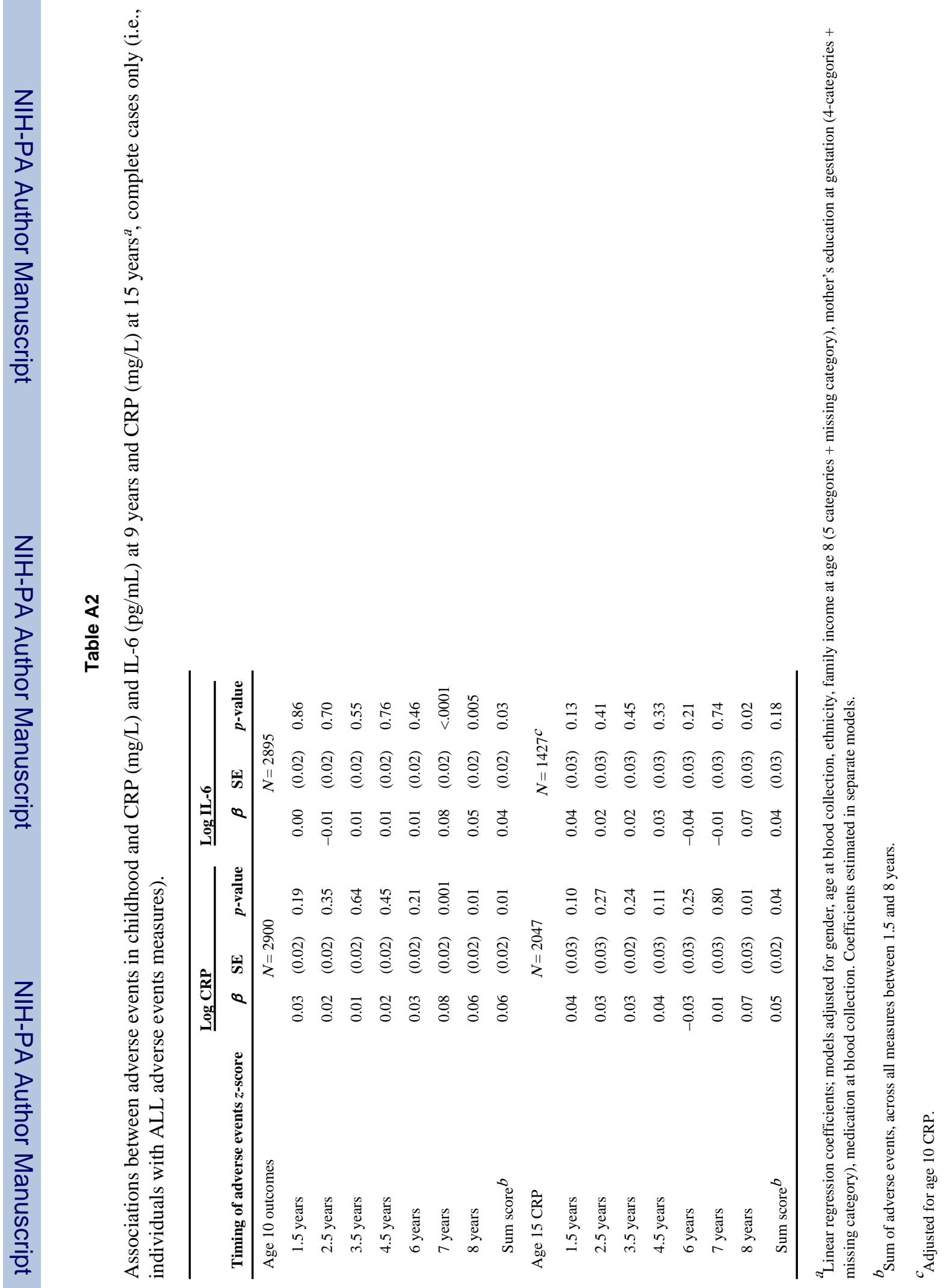

